\title{
Reflex Modification Audiometry: Assessment of Acoustic Sensory Processing in the Term Neonate
}

\author{
ENDLA K. ANDAY, MICHELLE E. COHEN, NANCY E. KELLEY, AND HOWARD S. HOFFMAN \\ Department of Pediatrics, University of Pennsylvania, School of Medicine, Philadelphia, Pennsylvania 19104 and \\ Department of Psychology, The Graduate School of Arts and Sciences, Bryn Mawr College, \\ Bryn Mawr, Pennsylvania 19010
}

\begin{abstract}
Behavioral and physiological work in animals and adult humans have established the sensitivity of various procedures and allowed delineation of the neuroanatomical pathways involved in sensory processing. Herein we used the glabellar reflex and reflex modification procedures to assess acoustic sensory processing capabilities in the term newborn infant. The eyeblink-eliciting device consisted of a miniature solenoid which could deliver a controlled tap. A photoreflective densitometer attached to a TDH-39 earphone assessed the eyeblinks. A total of 98 term infants was studied to determine how a response to a reflex-eliciting event (tap) was modified (either augmented or inhibited) by a mild exteroceptive stimulus (tone) which was presented at an appropriate lead interval. Ninety adult subjects were given identical testing procedures and their data were compared to that of the infants. The results of this study showed that newborn infants reliably exhibited an eyeblink response after a tap to the glabella. With fixed intensity tones, frequencies from 1 to $4 \mathrm{kHz}$ produced equivalent amounts of reflex augmentation in infants and adult subjects. Blink amplitude increased as a function of increased tap and tone intensity in both infants and adults. State change was shown to affect the amplitude of the reflexive eyeblink, but not the augmentation effect. However, neonates failed to show inhibition to either acoustic or tactile stimuli at an interstimulus interval that produced significant inhibition in the adult. These data indicate that reflex modification procedures provide an objective assessment of acoustic sensory processing in the term neonate. Further, they not only suggest that areas of the central nervous system that process sensory stimuli are not fully developed even at term birth, but that the neural system for augmentation and inhibition may be independent of each other. (Pediatr Res 23: 357-363, 1988)
\end{abstract}

Abbreviation

REM, rapid eye movements

Auditory signals may play an important role in the development of sensory and perceptual processes as well as form the basis for communicative behavior (1). However, the study of auditory development in the human infant has been hampered by a lack of both quantifiable and reliable data. Various methods used to determine hearing in the infant have included physiolog-

Received August 19, 1987; accepted December 1, 1987.

Correspondence Endla K. Anday, Department of Pediatrics, 2 Maloney Building, Hospital of the University of Pennsylvania, 36th and Spruce Street, Philadelphia, PA 19104.

Supported by NIH Grant HD 10511. ical and electrophysiological measures, reflex behavior, and learning procedures. Not all of these methods are useful at all ages; not all provide objective measurement of auditory perception.

The purpose herein was to investigate the applicability of a psychophysical approach, based on behavioral phenomenon called reflex modification, to objectively assess acoustic sensory competence in the term infant $(2,3)$. This testing procedure originated in the observation that a variety of auditory and nonauditory stimuli consistently evoked a startle reflex in both humans and animals, the most constant component of which was the eyeblink (4-7). Subsequent investigation established that the elicited reflex could be modulated in amplitude by a variety of sensory stimuli and that the changes in eyeblink response produced by these stimuli could be monitored by measuring the amplitude of the blink component of the reflex $(8,9)$. Our study used a tone as a reflex-modifying event and a tap to the glabella as the reflex-eliciting stimulus. By varying the characteristics of the tone and its temporal relationship to the tap, and by measuring the amplitude of the resulting eyeblink, the limits of several reflex modifying procedures on the auditory system in the neonate could be objectively assessed and provide insight into the way in which the central nervous system processes acoustic signals of the sort that form the elements of human speech. Further, if the identical procedures were applied to an adult subject, one could determine whether and how reflex modification effects change in the course of development.

Our study was designed to answer the following questions: 1) Does the glabellar tap reliably elicit a blink response in the term newborn infant; is the response modified by tap intensity or state change? 2) How sensitive is the term neonate to reflex modification procedures; specifically, is the response affected by tone intensity, tone frequency, state change, or interstimulus interval? 3) How does the response of the term neonate to reflex modification procedures compare with the adult and what are the implications?

\section{METHODS}

Study population. Infants. A total of 98 infants $(58$ females, 40 males) ranging in postnatal age from 12 to $62 \mathrm{~h}$ (mean $291 / 2$ h) were tested. Selection of an infant required an uncomplicated term gestation (range 37-42 wk, mean $39.0 \mathrm{wk}$ ) with a 1 and 5 min Apgar score of $>8$, and whose birth weight was appropriate for gestational age. Inclusion into the study required a normal pediatric examination, including an otological exam.

Adults. Ninety adult volunteers were recruited and ranged in age from 18 to $35 \mathrm{yr}$.

Apparatus. Infants were tested in a room separate from the nursery, with an ambient noise level of approximately $55 \mathrm{dBA}$ Adults were tested in a double-wall, sound-treated chamber with an ambient noise level below $25 \mathrm{dBA}$. 
Figure 1 illustrates the devices used to elicit and detect eyeblinks. The eyeblink-eliciting device consisted of a miniature light-weight solenoid (Guardian electric TP 3.5 × 9-1-24 V DC). A rounded Teflon striker $(1 \mathrm{~cm}$ in diameter and $5 \mathrm{~mm}$ in height $)$ was attached to its plunger and a miniature compression spring was used to keep the plunger in a retracted position during the interval between taps. When the coil of the solenoid was activated by a brief $(50 \mathrm{~ms})$ pulse of direct current, the plunger and Teflon striker were thrust forward with an impulse (in newtons/s) that as measured with a ballistic pendulum was directly proportional to the applied voltage. The power source activating the solenoid produced voltages of 8 to a maximum of $20 \mathrm{~V} \mathrm{DC}$. By positioning this device over the glabella and pressing an external switch, a stimulus-eliciting event could be initiated.

The eyeblink-assessing device consisted of a miniature photoreflective densitometer manufactured by Coulbourn Instruments. In this optical monitor, the light source was an infrared light-emitting diode, incorporated in the transducer assembly so that illuminance was independent of head position. This was pointed toward the eye approximately $25 \mathrm{~mm}$ from the upper lid. A phototransistor completely covered with an infrared filter that passed wave lengths greater than $850 \mathrm{~nm}$ (Kodak Wratten gelatin filter $87 \mathrm{C}$ ) detected the reflected signal. The output of the LED was modulated to render the device insensitive to ambient light; the output of the phototransistor was in turn filtered so that low-frequency components (e.g. $60 \mathrm{~Hz}$ and its harmonics) were rejected. The sensitivity of this device compares favorably with that of a low-torque potentiometer which is most commonly used to measure eyeblinks, and detects movement as small as 33 $\mu \mathrm{m}$ and latency to an accuracy of $\pm 1 \mathrm{~ms}$.

Detailed studies of the human blink reflex using electrophysiological measurements have determined the presence of two components of the blink reflex. The second component, evaluated herein has a latency of approximately $36 \mathrm{~ms}$ from the onset of the glabellar tap, and a duration of 30 to $40 \mathrm{~ms}(10)$. The

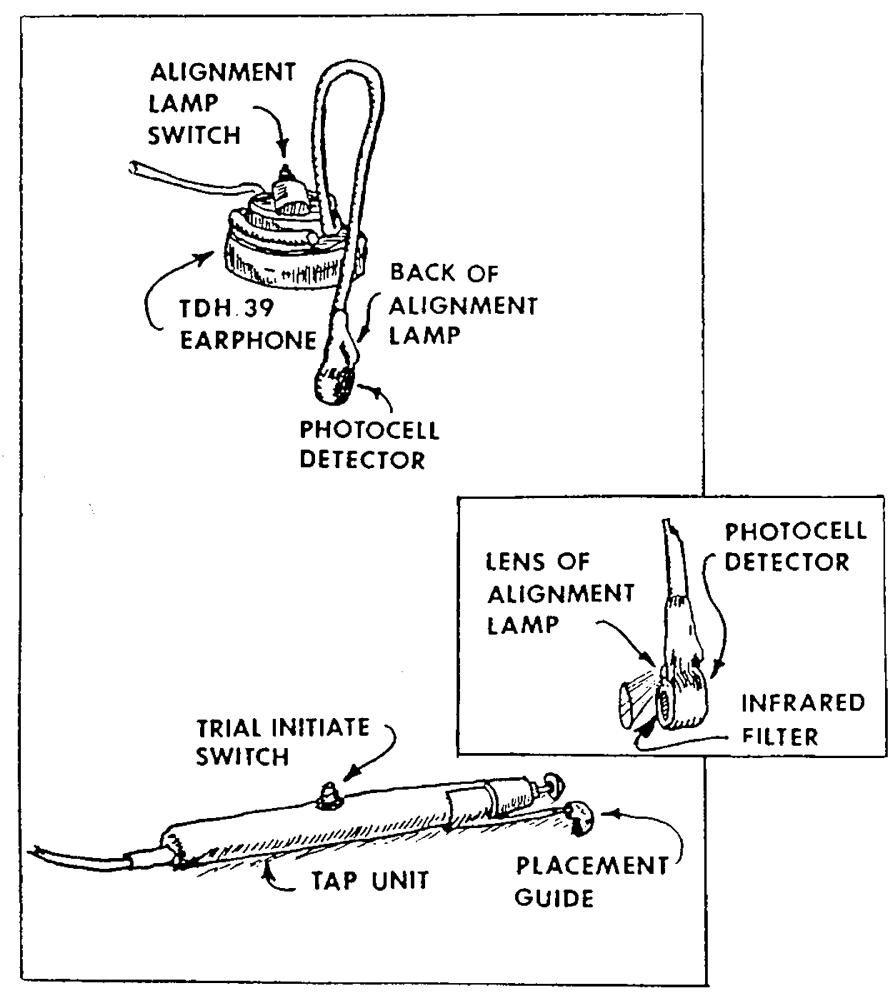

Fig. 1. Eyeblink eliciting and testing device for neonates. The blinkmonitoring device was mounted on an earphone. The tap unit that was positioned at the glabella consisted of a solenoid with a Teflon striker mounted in a small flashlight housing. Adults were tested with similar equipment. computer was programed to score the peak movement of the eyeblink amplitude within a predetermined window. With the arrangement herein, response amplitude was defined as the peak output of the eyeblink-monitoring device during the 150 -ms period that began with the onset of the tap to allow for adequate time from initiation of the stimulus to the response so as to exclude less than $1 \%$ of movements that followed a reflex stimulus. The output (in V) was read by a peak detector (Coulbourn module S76-31). For adult subjects, the output of the peak detector was digitized and strobed to a TRS-80 Radio Shack computer; for infants, it was sent to a digital voltmeter. A TDH39 earphone was attached to the stalk carrying the blink detector. The acoustic stimuli, which served as reflex modifying signals, were pure tones generated, shaped, amplified, and timed by Coulbourn modules and presented monaurally through the earphone.

Before the start of each testing session, the measuring devices and stimuli (tones) were calibrated with a General Radio precision sound-level meter (model 1561-A) fitted with a P-7 microphone and an ANSI-type coupler to allow proper function and safe levels of the stimuli. Additional ensurance was provided by the fail-safe design of the power supplies and by the use of an inline fuse in the tap unit.

Procedure. The study was reviewed and approved by the University of Pennsylvania Committee on Research Involving Humans. After obtaining parental consent, and approximately 30 min after a feeding, the sleeping infant was moved into a separate room. Infant state was rated on a 7-point scale (11) and all trials were initiated by placing the tap delivery unit over the infant's glabella by experimenter 1 . A second experimenter operated the programing apparatus so the first experimenter was unaware of the condition that prevailed on a given trial. This general procedure was followed for all of the infant testing presented herein.

Adults were tested with the same stimuli as the infants; the programing was automatic and run by the computer. After securing written agreement to be tested, adult subjects were escorted into the test chamber and fitted with a headband containing the tap delivery and eyeblink detection unit and earphones.

For each of the following experiments, 15 infants and 15 adults were tested, with the exception of study 5 in which eight infants were studied.

Experiment 1 -blink at various tap intensity. The first study examined the way blink amplitude in the sleeping newborn varied as a function of tap intensity. It was essential to establish whether our procedure was adequate to detect tap intensity effects in order to proceed to an analysis of reflex modification variables.

Each infant was exposed to four different tap intensities: 8,12 , 16 , and $20 \mathrm{~V} \mathrm{DC}$, with a duration of $50 \mathrm{~ms}$ for each tap. There were 12 blocks of four trials with each of four tap intensities presented in random order within each trail block. Taps occurred at an interval of $20 \pm 5 \mathrm{~s}$, with a single session lasting approximately 20 min. Adult subjects were tested with identical stimuli.

Reflex modification experiments. The next several studies examined the phenomenon of reflex modification using a tap and tone paradigm (Fig. 2). The two major processes of reflex modification are reflex augmentation and inhibition. Augmentation can be defined as the process of enhanced reflex amplitude due to the concurrent presentation of a weak sensory event and reflex-eliciting stimulus (Fig. $2 b$ ); inhibition is the process of decreased reflex amplitude due to the presentation of a sensory event at an optimal lead interval before the reflex-eliciting stimulus (Fig. 2c). Specifically, we assessed the ability of the newborn infant to augment and/or to inhibit the eyeblink response when a tone was presented simultaneously or before the glabellar tap, respectively. The results obtained in the neonates were compared to the results in adults.

Experiment 2-blink at various tone frequency. This study was designed to examine the effect of tone frequency on the reflex 


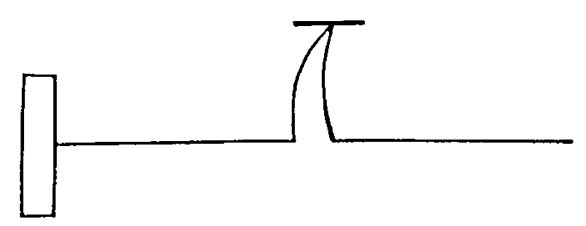

GLABELLA REFLEX

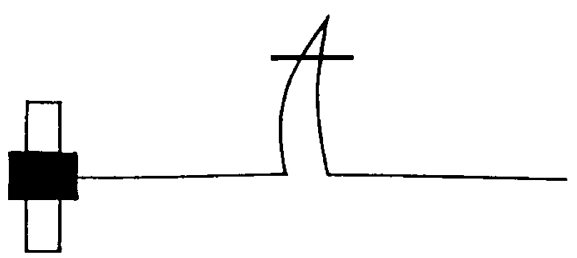

AUGMENTATION

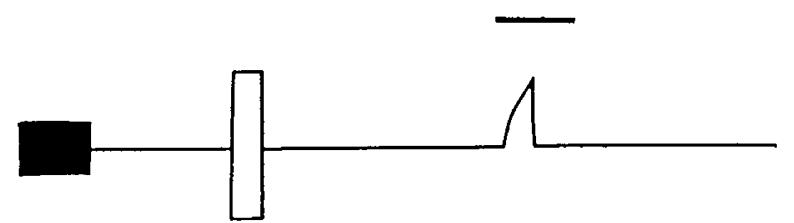

INHIBITION

Fig. 2. Schematized representation of reflex modification paradigm. The baseline glabellar tap is illustrated in $a$. Augmentation is depicted as a tone presented simultaneously with the glabellar tap and results in increased eyeblink amplitude $(b)$. Inhibition is represented as a tone or tap preceding the glabellar tap at a given lead interval and results in decreased eyeblink amplitude $(c)$.

augmentation paradigm. For infants, as for adults, there were two basic conditions: 1) tap only-16 V DC, $50 \mathrm{~ms}$ tap to the glabella with no concurrent acoustic stimulus and 2) tap with a simultaneously presented $90 \mathrm{~dB}$ SPL (re: $0.0002 \mathrm{dyne} / \mathrm{cm}^{2}$ ) tone of $50 \mathrm{~ms}$ duration, $5 \mathrm{~ms}$ rise/fall time. On a given tap plus tone trial, the tone frequency could be either 1,2, or $4 \mathrm{kHz}$. Each of four trials was presented in random order within each of 12 blocks of trials for a total of 48 trials.

Experiment 3-blink at various tone intensity. The next study examined details of reflex augmentation as a function of the intensity of a reflex-augmenting acoustic signal. Infants and adults were tested on two conditions: 1) tap only as in experiment 2 and 2) tap with a simultaneously presented $1 \mathrm{kHz}(50 \mathrm{~ms}$ duration, $5 \mathrm{~ms}$ rise/fall time) tone at one of four intensities, 60 , 70,80 , and $90 \mathrm{~dB}$ SPL. With this arrangement, a total of five trial conditions within each of 12 blocks of trials for a total of 60 trials was presented in random order.

Experiment 4-blink at various tap and tone intensity. This next study examined the effect of varying tap intensity on the amount of reflex augmentation afforded by the simultaneous presentation of a tone. Infants and adults were tested on two stimulus conditions: 1) tap only with tap intensity of either 8 or $14 \mathrm{~V} \mathrm{DC}$ and 2) tap with a simultaneously presented $1 \mathrm{kHz}, 90$ $\mathrm{dB}$ SPL tone at the two tap intensities. A sequence of four trial conditions were presented randomly within each of 10 blocks for a total of 40 trials.

Experiment 5-blink at various state change. This study attempted to determine how reflex elicitation and augmentation might vary as a function of sleep state in the newborn infant. Specifically, the responses to tap alone (16 V DC) and to tap with an augmenting 70 or $90 \mathrm{~dB}$ SPL tone was administered to infants who vacillated between quiet sleep (stage 1) and active sleep (stage II). Infants received at least two of each kind of trial in each of the two states.

Experiment 6-blink at various interstimulus interval (tone). This next study was designed to examine eyeblink response amplitude as a function of interstimulus interval using a reflex inhibition paradigm. Infants and adults were tested on two conditions: 1) tap only-16 V DC and 2) tap preceded by acoustic stimulation $-1 \mathrm{kHz}, 90 \mathrm{~dB}$ SPL tone preceded the onset of the blink-eliciting tap by one of four intervals, 75, 150,300, or $600 \mathrm{~ms}$. A total of five trials was run presented in random order within each of 10 trial blocks for a total of 50 trials.

After the experimental trials in studies 2 through 6, each subject received five trials of a $90 \mathrm{~dB}$ SPL $1 \mathrm{kHz}$ tone without accompanying or subsequent tap to the glabella to determine if the tones used were capable alone of eliciting measurable blinks. For infants and adults, no blinks ever occurred to the tone only control trials at the end of each session.

Experiment 7-blink at various interstimulus interval (tap). The final study was designed to determine if a nonauditory stimulus might induce reflex inhibition. For infants and adults a trial consisted of a $14 \mathrm{~V} \mathrm{DC} 50 \mathrm{~ms}$ tap to the glabella followed at a given interval by a second tap of the same intensity and duration. Four intertap intervals used were: $300,600,900$, and $1200 \mathrm{~ms}$ as measured from the onset of the first to the onset of the second tap. Trials of eight blocks for a total of 32 trials were given in which each of the four conditions was presented in random order.

Statistical analyses to determine between and within group differences for each experiment were performed using analysis of variance for repeated measures and Newman-Keuls tests where statistical significance was demonstrated. Values are expressed as mean \pm SEM.

\section{RESULTS}

Experiment 1-blink at various tap intensity. Figure 3 shows the mean eyeblink amplitude elicited in infants and adults by each of the four tap intensities studied. Two-way analysis of variance showed that for all tap intensities $>8 \mathrm{~V}$, infants had a significantly smaller $(p<0.01)$ eyeblink response compared to adults. Adult subjects exhibited significantly $(p<0.01)$ larger blinks with each increased tap intensity. While there was a trend toward increased eyeblink amplitude as tap intensity increased in the infants, the differences were not significant.

Experiment 2-blink at various tone frequency. The mean

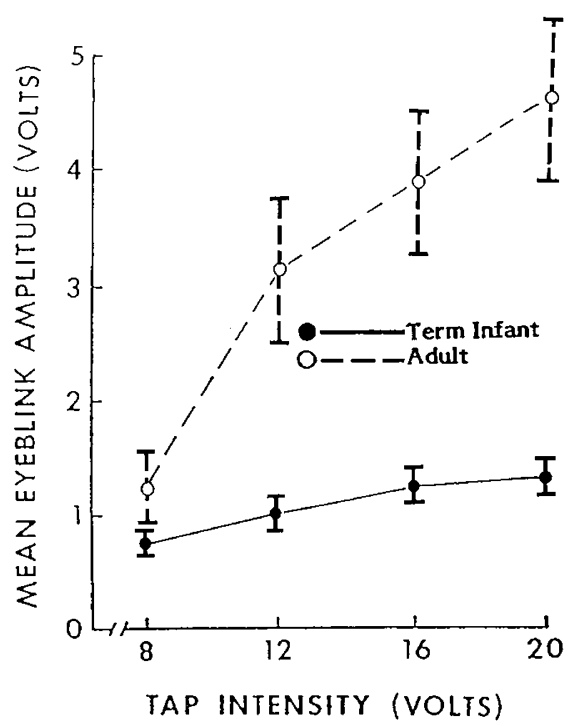

Fig. 3. Mean amplitude \pm SEM of tap-elicited eyeblinks in infants ( $n$ $=15)$ and adults $(n=15)$ as a function of tap intensity. 
amplitude of the eyeblinks elicited in neonates and adults when tone frequencies were varied is shown in Figure 4 . Both infants and adults exhibited reflex augmentation to each of the three tone frequencies when compared to the $16 \mathrm{~V}$ tap alone $(p<$ 0.01 ). A two-way analysis of variance with repeated measures on the conditions factor was performed on the combined data from both groups of subjects and showed no difference in response between infants and adults. Subsequent separate Newman-Keuls tests revealed that in both infants and adults, reflex augmentation did not vary with the frequency of the tones used, but rather augmentation occurred as a result of the tone itself.

Experiment 3-blink at various tone intensity. The effect of tone intensity is shown in Figure 5. The mean eyeblink amplitude elicited in neonates and adults on each of five kinds of trials using four tone intensities demonstrated that both neonates and adults exhibited an increased blink as tone intensity increased ( $p$ $<0.01$ ). Using simple main effects to assess statistical significance of the individual comparisons, significant $(p<0.05)$ augmentation occurred only at the $90 \mathrm{~dB}$ level.

Experiment 4-blink at various tap and tone intensity. Figure 6 shows the mean eyeblink amplitude elicited in neonates and adults on each of four kinds of trials, varying tap intensity with identical tone. Both infants and adults showed augmentation to each of the two tap intensities when the tap was accompanied by the $90 \mathrm{~dB}$ tone. A three-way analysis of variance with repeated measures on two condition factors was performed on the data. There was a significant effect of tap intensity $(p<0.01)$ and of tone presentation $(p<0.01)$ in both as well as an overall significant $(p<0.01)$ difference of response between infants versus adults. However, no significant interactions between infants and adults and tap intensity or tone presence was seen nor was the three-way interaction significant.

Experiment 5-blink at various state change. The effect of state change in reflex augmentation is shown in Figure 7. Mean eyeblink amplitude elicited the neonate using a tap alone and a tap with an augmenting 70 or $90 \mathrm{~dB}$ tone during quiet (stage I) or active (stage II) sleep was examined. Infants were less responsive to the study conditions when in quiet sleep than when in

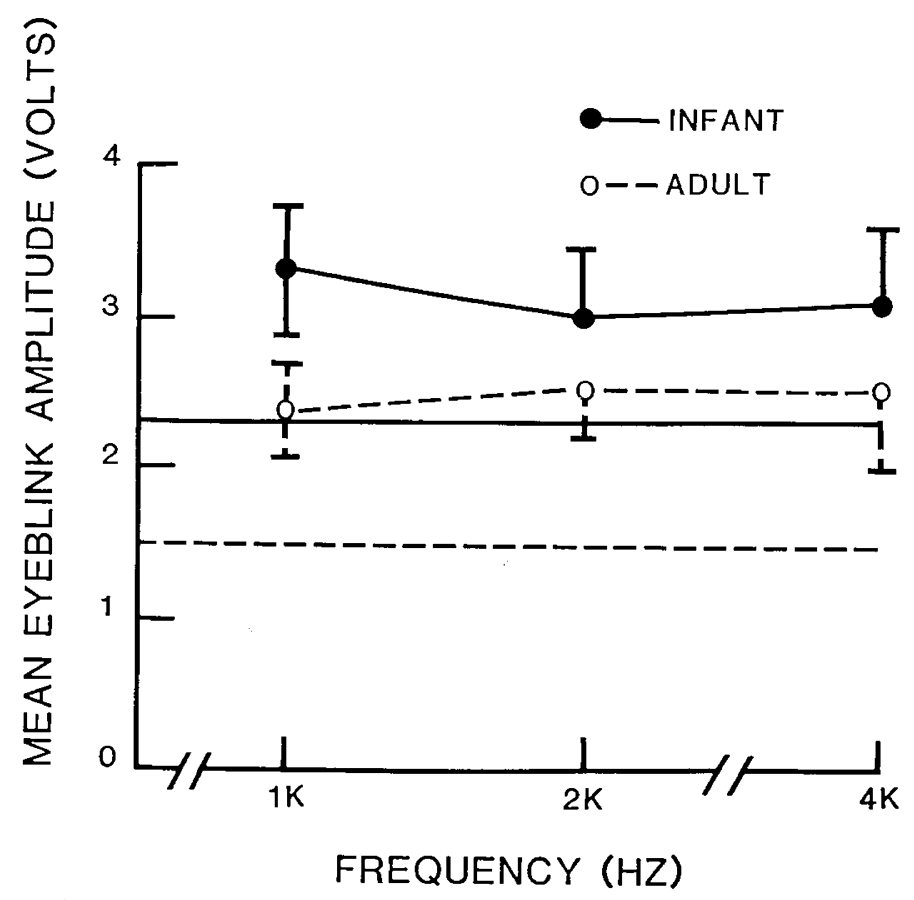

Fig. 4. Mean amplitude \pm SEM of tap-elicited eyeblinks as a function of tone frequency presented simultaneously with a tap in infants and adults. The solid and dashed horizontal lines indicate the mean amplitude response without a simultaneously presented tone in infants $(n=15)$ and adults $(n=15)$, respectively. The SEM of the baseline tap for infants and adults are \pm 0.30 and \pm 0.28 , respectively.

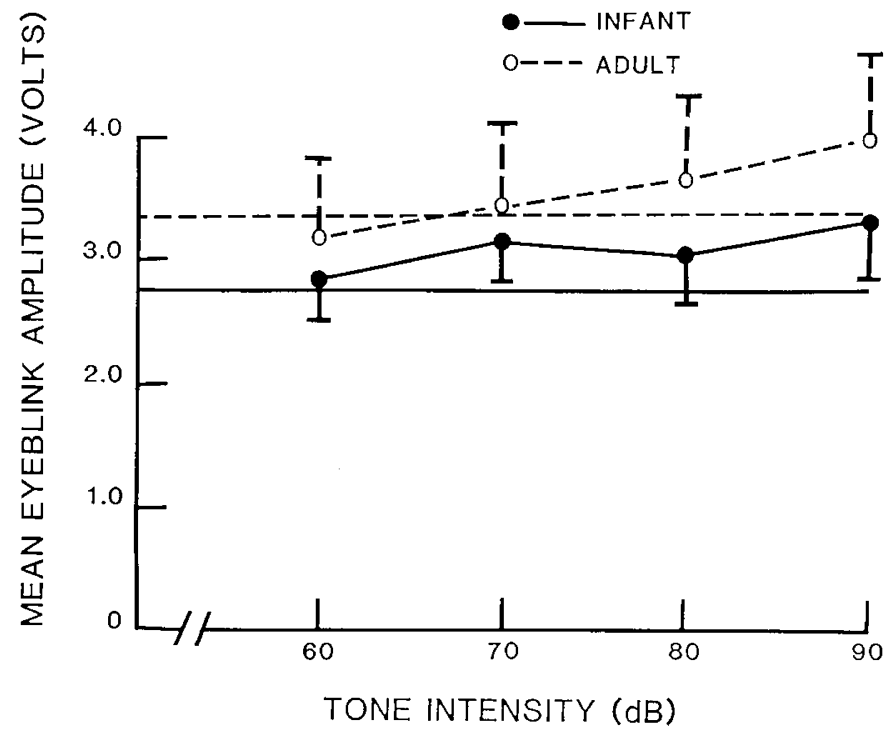

Fig. 5. Mean eyeblink amplitude \pm SEM elicited by a tap when tones of various intensities were presented simultaneously with the tap in infants and adults. The solid and dashed horizontal lines indicate the mean amplitude response without a simultaneously presented tone in infants $(n=15)$ and adults $(n=15)$, respectively. The SEM of the baseline tap for infants and adults are \pm 0.21 and \pm 0.61 , respectively.

active sleep. A two-way analysis of variance with repeated measures showed significant effect of state change on eyeblink amplitude $(p<0.01)$ and tone presentation $(p<0.05)$ but no interaction between these factors. These results indicate that sleep state plays a role in the overall level of the responses elicited during the tests but state change does not interfere with the reflex augmentation effect.

Experiment 6-blink at various interstimulus interval (tone). Figure 8 shows the mean eyeblink amplitude elicited in neonates and adults using a tap after a tone at various intervals. Infants failed to show evidence of inhibition; adults exhibited significant $(p<0.01)$ inhibition at each of the four lead times tested. A twoway analysis of variance showed a significant $(p<0.01)$ difference between infants and adults in eyeblink amplitude response to a tone prestimulus at all interstimulus intervals.

Experiment 7-blink at various interstimulus interval (tap). The failure to obtain reflex inhibition in neonates in experiment 6 suggested that perhaps infants did not process tone as a reflexinhibiting stimulus. We speculated that by using a nonauditory stimulus such as a tap, as both the reflex-inhibiting and reflexeliciting stimulus, we could be certain that the signal had been detected. A three-way analysis of variance showed significant ( $p$ $<0.01$ ) interaction between group, interstimulus interval, and tap position (Fig. 9). Simple main effects showed a significant difference on the effect of interstimulus interval between adults and infants $(p<0.05)$. For adults there were significant differences between the first and second tap at all interstimulus intervals $(p<0.05)$ while infants showed significant differences only between the first and second tap at intertap intervals at 600 ( $p$ $<0.05), 900(p<0.05)$, and $1200 \mathrm{~ms}(p<0.01)$.

\section{DISCUSSION}

Methodology permitting objective examination of the response to auditory stimuli in human infants is limited as the majority of testing procedures require a cooperative subject. A psychophysical approach, based on behavioral phenomenon called reflex modification, has been shown to yield objective information about the processing of acoustic signals. Reflex modification, the procedure used herein, had its origin more than $20 \mathrm{yr}$ ago in a series of investigations in animals of the manner in which an overt startle reflex elicited by an intense noise could be modified 


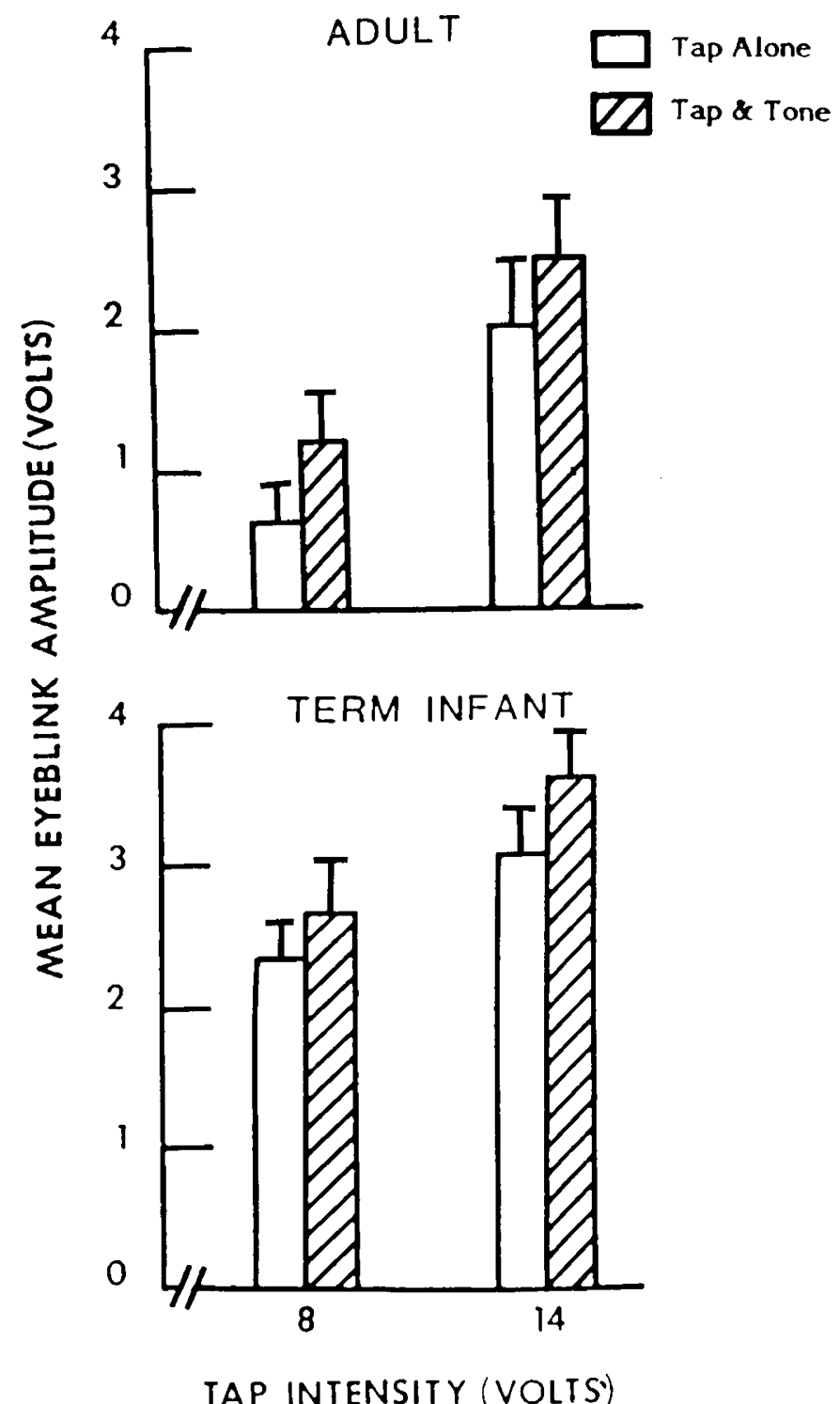

Fig. 6. Mean amplitude \pm SEM of tap-elicited eyeblinks as a function of varying tap intensity to the glabella accompanied by identical tone in infants $(n=15)$, and adults $(n=15)$.

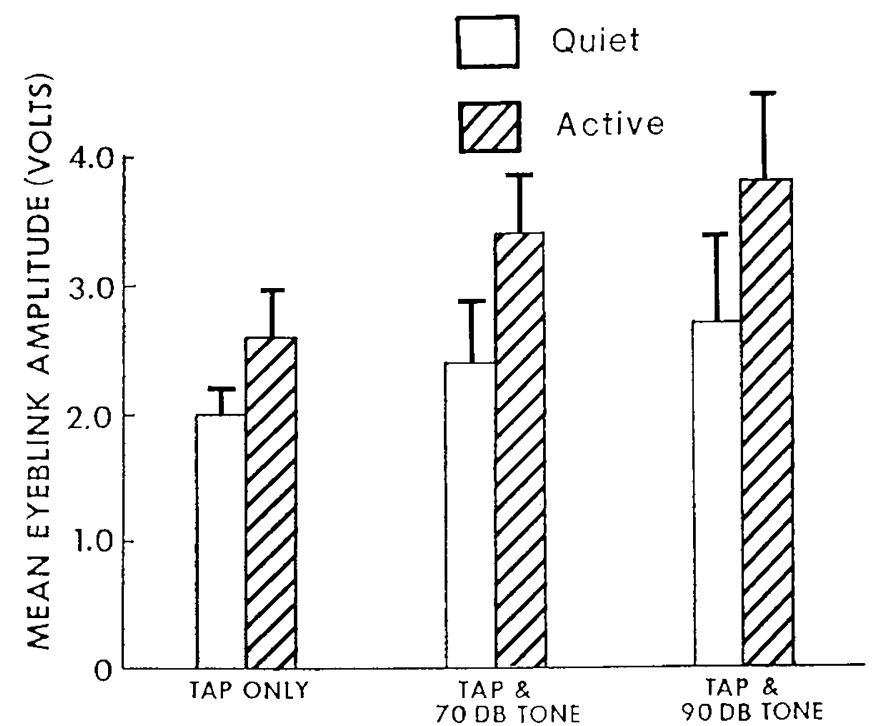

Fig. 7. Effect of sleep state on eyeblink amplitude when tone intensities of 70 or $90 \mathrm{~dB}$ SPL accompanied the tap in infants $(n=8)$. Values are mean \pm SEM.

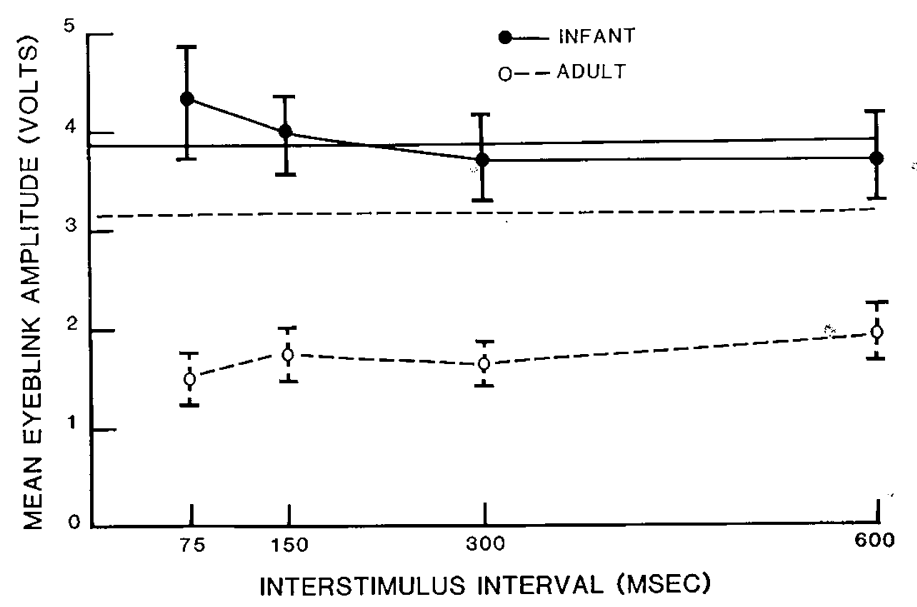

Fig. 8. Mean eyeblink amplitude \pm SEM when tone preceded the tap by various intervals in infants and adults. The solid and dashed horizontal lines represent the mean eyeblink amplitude without a simultaneously presented tone in infants $(n=15)$ and adults $(n=15)$, respectively. The SEM of the baseline tap for infants and adults are \pm 0.68 and \pm 0.31 , respectively.

by the presentation of a weaker sound either preceding or accompanying the reflex-eliciting event $(2,12)$ This effect was found to be robust and independent of associative or conditioning processes $(13,14)$. Further investigations established the startle-eliciting stimulus and reflex-modifying signal need not be in the same sensory modality (8). Studies in adult humans delineated that reflex modification effects could be found if the eyeblink rather than skeletal startle response was measured and that a mild tap to the glabella reliably elicited a blink response. The sensitivity of this procedure was confirmed when tones as low as $30 \mathrm{~dB}$ SPL preceded or accompanied a tactile eyeblink-eliciting stimulus and led to the speculation that a similar paradigm could be used to assess acoustic sensory processing in the human infant (9).

The blink reflex elicited by the glabella tap in infants $<3$ days of age in our study suggests the central reflex arc is established by term birth. This is supported by the findings of Hooker (15) and Humphrey and Hooker (16) who showed that reflexive behavior could be demonstrated in the human fetus as early as $71 / 2$ wk gestation when surface areas of the face innervated by the sensory fibers of the trigeminal nerve were stimulated, resulting in a reaction characterized by a total pattern response (17). At 10-11 wk gestation, an exteroceptive stimulus over the upper eyelid resulted in a local reflex characterized by contraction of the ipsilateral orbicularis oculi. By 32-34 wk gestation, a tap to the glabella elicited an eyeblink response (18). There are two components of the blink response: the first, an oligosynaptic reflex arc results in contraction of the ipsilateral orbicularis oculi muscle $(19,20)$; the second, a polysynaptic reflex are, which was evaluated herein, has a long afferent limb mediated by fibers of the ipsilateral spinal trigeminal tract. These fibers project on the spinal trigeminal nucleus in the dorsal medulla. From here, fibers project rostrally ipsi- and bilaterally via the propriobulbar fiber system of the lateral reticular formation to innervate the facial nuclei $(21-23)$. The glabellar reflex thus is an unlearned expression of the functional capacity of an individual and depends on the maturation of an inherited neural mechanism.

Whereas both infants and adults herein exhibited increased eyeblink amplitude in response to increased tap intensity, infants as a whole were not as responsive and their intensity function was not as steep as the adult subjects. Previous studies of reflex responses from the orbicularis oculi muscle after electirisal stimulation of the supraorbital nerve in term infants $<3$ days of age showed the mean amplitude of response was reduced and latency was significantly greater in infants as compared to adults (6). This may be due to decreased conduction velocity secondary to 

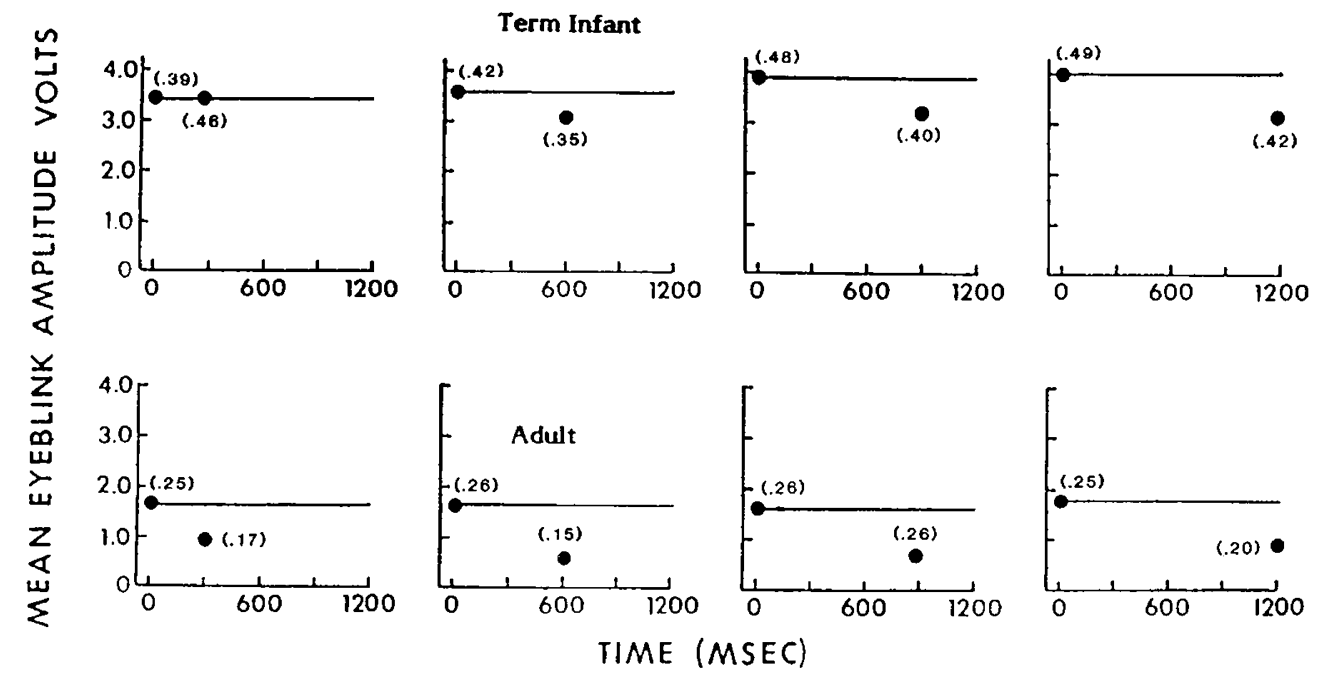

Fig. 9. Mean eyeblink amplitude \pm SEM elicited by an identical tap in a pair at various intertap intervals $(I S I)$ in infants $(n=15)$ and adults $(n$ $=15)$. The solid horizontal line represents the first of the pair of taps at each lead interval. The numbers in parentheses signify SEM at each intertap intervals.

incomplete myelination of the trigeminal and facial nerves as well as decreased synapses between interneurons in the brainstem pathway involved in this reflex arc (24). An alteration in sleep state may also account for differences in response between infants and adults (25). We observed a decreased eyeblink amplitude in quiet (non-REM) as compared to active (REM) sleep in the infants. Vecchierine-Blineau and Guiheneuc (26) showed that different states of waking and sleeping in adult and infant subjects influenced the second component of the blink reflex, causing a more pronounced depressed response during quiet sleep in the infant, and suggested the polysynaptic reflex arc may be subject to an inhibitory influence at the level of the reticular formation during non-REM sleep.

The augmented response we observed while varying tone intensity and frequency indicates the newborn infant is able to process acoustic tones within the same intensity range as the adult. This is in contrast to studies by Eisenberg (27) and Schneider et al. (28) in which newborns were noted to have a decreased sensitivity in hearing relative to adults, in the first few days of life. Herein we suggest that, at least with pure a tone stimulus in the frequency range of 1000 to $4000 \mathrm{~Hz}$, the term newborn does not demonstrate an increased hearing level at the tone intensities studied. This finding is in agreement with Engel and Young (29) who demonstrated that hearing level in the neonate was within $15 \mathrm{~dB}$ of adult norms at all frequencies tested. The discrepancies that exist between various studies using pure tones may be due to the rise/fall time of the pure tone. We used a $5 \mathrm{~ms}$ rise/fall time for the sound stimulus that in effect resulted in an increased band width and stronger signal. Further, our results are consistent with observations from the laboratory of Graham et al. (12) that infants, relative to adults, are more reactive to conditions that facilitate responding.

In contrast to reflexive augmentation in response to acoustic signals, term infants failed to exhibit inhibition to tones under conditions that elicited robust inhibition in adult subjects. This would suggest the neural systems responsible for reflex augmentation and inhibition are in some measure independent of each other (13), and the neural pathway for inhibition may not be fully developed at term birth. Previous investigation in human infants using a tone/tap paradigm to test inhibition, but at a lead interval more than $200 \mathrm{~ms}$, found weak reflex inhibition responses in 6- to 9-wk-old infants; significant inhibition close to the interval optimal for adult inhibition was not attained until 24 wk postnatally (12). Graham et al. (12) have suggested the underlying mechanism for this effect may be due to presynaptic inhibition resulting from partial depolarization of flexor reflex afferents by an acoustic prestimulus and that this system is not fully mature in the infant $<6$ months of age.

The observation herein that a tap, rather than a tone stimulus, with an intertap interval of at least $600 \mathrm{~ms}$ was sufficient to produce reliable inhibition in the neonate suggests the acoustic inhibition reflex arc involves a more complex circuit than the tactile inhibition paradigm and that given a strong enough stimulus and long enough lead interval, the behavioral mechanisms responsible for the inhibition effect are functional at term birth, but slow to act. Support for the proposition that the neural system for reflex inhibition is not fully developed at birth is provided by Parisi and Ison's (30) investigation in newborn animals. In this study using tactile and auditory prestimuli in the neonatal rat, inhibition developed gradually after birth, with tactile stimuli providing reliable inhibition at an earlier age than acoustic stimuli. The difference between the time of onset of inhibition for these two modalities indicates the importance of the maturation of the sensory systems. Further, it supports the observation herein of the later maturation in either the intrinsic auditory structures or in the connections between these structures and the common substrate for reflex control. Although the exact pathway for the acoustic startle reflex is not known in man, based on investigations in animals, the areas probably involved in acoustic augmentation and inhibition are depicted in Figure $10(7,31-33)$. The pathway for auditory inhibition of the acoustic startle reflex appears to involve areas of the midbrain shared by the neural structures mediating augmentation, but includes projections from the inferior colliculus to the nucleus cuneiformis and nucleus parabrachialis ventralis in the lateral tegmental area (34). In the human infant, marked functional changes occur in these locations of the central nervous system during perinatal development to include: myelination and growth of axons, multiplication and extension of dendrites, synaptic development, and synthetic enzyme activity.

In summary, the results herein would seem to have several implications for an understanding of sensorineural competence in the neonate. By demonstrating reliable reflex augmentation, there is objective indication that tones are at some level being processed. Further, our results indicate that the neural system for inhibition is structurally established but functionally immature, even at term birth. By using reflex modification procedures one can achieve a better understanding of central nervous system function and organization; how atypical early experiences such as prematurity, asphyxia, and other factors are expressed in the altered development of brain activity becomes even more important if the specific reasons for the more subtle effects seen in 


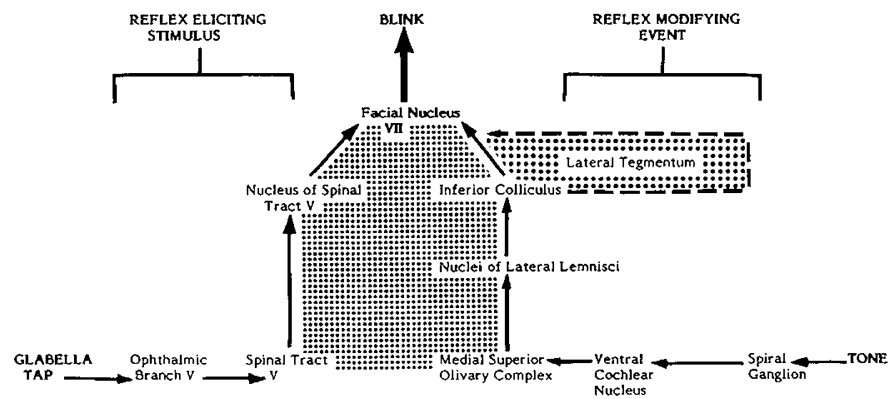

Fig. 10. Proposed neural structures mediating augmentation and inhibition of the glabellar tap/acoustic reflex arc in the human. The solid line represents the shared pathway for modulating the augmentation and inhibition responses. The dashed line signifies the descending projections from the inferior colliculus to the lateral pons that are involved in auditory reflex inhibition. The stippled area represents the reticular formation.

perception and cognitive disorders at later stages are to be understood.

Acknowledgments. The authors thank Lynn Grossman, R.N., for assisting in testing the infants and Marian Bergan for the preparation of this manuscript.

\section{REFERENCES}

1. Aslin RW, Pisoni DB, Jusczyk PW 1971 Auditory development and speech perception in infancy. In: Haith MM, Campos JJ (eds) Infancy and Developmental Psychobiology. Handbook of Child Psychology, Vol 2. (Mussen PH ed). John Wiley, New York, pp 573-687

2. Hoffman HS, Ison JR 1980 Reflex modification in the domain of startle: I Some empirical findings and their implications for how the nervous system processes sensory input. Psychol Rev 87:175-189

3. Hoffman HS, Searle $J 1968$ Acoustic and temporal factors in the evocation of startle. J Acoust Soc Am 43:269-282

4. Jacobson E 1926 Response to a sudden unexpected stimulus. J Exp Psychol 9:19-25

5. Landis C, Hunt WA 1936 Studies of the startle pattern. III. Facial pattern. J Psychol 2:215-219

6. Kimura J, Bodensteiner J, Yamanda T 1977 Electrically elicited blink reflex in normal neonates. Arch Neurol 34:246-249

7. Leitner DS, Powers AS, Hoffman HS 1980 The neural substrate of the startle response. Physiol Behav 25:291-297

8. Buckland G, Buckland J, Jamieson C, Ison JR 1969 Inhibition of the startle response to acoustic stimulation produced by visual prestimulation. J Comp Physiol Psychol 67:493-496

9. Stitt CL, Hoffman HS, DeVido CJ 1980 Modification of the human glabella reflex by antecedent acoustic stimulation. Percept Psychophys 27:82-88
10. Shahani BT 1981 The human blink reflex. J Neurol Neurosurg Psychiat 33:792-800

11. Anders T, Emde R, Parmelee A 1971 A Manual of Standardized Terminology Techniques and Criteria for Scoring States of Sleep and Wakefulness in Newborn Infants. Brain Information Service, UCLA School of Health Services, Los Angeles

12. Graham FK, Strock BD, Ziegler BL 1981 Excitatory and inhibitory influences on reflex responsiveness. In: Collins WA (ed) Aspects of the Development of Competence. Erlbaum, Hillside, NJ

13. Stitt CL, Hoffman HS, Marsh RR 1976 Interactive versus independence of startle-modification process in the rat. J Exp Psychol Animal Behavior Proc 2:260-265

14. Hoffman HS, Cohen ME, Stitt CL 1981 Acoustic augmentation and inhibition of the human eyeblink. J Exp Psychol 7:1357-1362

15. Hooker D 1960 Development reaction to environment. Yale J Biol Med $32: 431-440$

16. Humphrey T, Hooker D 1959 Double simultaneous stimulation of human fetuses and the anatomical patterns underlying the reflexes elicited. J Comp Neurol 112:75-102

17. Coghill GE 1929 Anatomy and the Problem of Behavior. Cambridge University Press, Cambridge, England

18. Robinson RJ 1966 Assessment of gestational age by neurological examination Arch Dis Child 41:437-447

19. Tackmann W, Ettlin T, Barth R 1982 Blink reflexes elicited by electrical, acoustic and visual stimuli. Eur Neurol 21:210-216

20. Trontelj MA, Trontelj JV 1978 Reflex arc of the first component of the human blink reflex. J Neurol Neurosurg Psychiat 41:538-547

21. Kugelberg E 1951 Facial reflexes. Brain 75:385-396

22. Ongerboer de Visser BW, Kuypers HGJM 1978 Late blink reflex changes in internal medullary lesions. Brain 101:285-294

23. Rushworth G 1962 Observations on blink reflexes. J Neurol Neurosurg Psychiat 25:93-108

24. Dobbins J, Sands J 1973 Quantitative growth and development of the human brain. Arch Dis Child 48:757-767

25. Silverstein LD, Graham FK, Calloway JM 1980 Preconditions and the excitability of the human orbicularis oculi reflex as a function of state. Electroencephalogr Clin Neurophysiol 48:406-417

26. Vecchierini-Blineau ME, Guiheneuc P 1984 Maturation of the blink reflex in infant. Eur Neurol 23:449-458

27. Eisenberg RB 1976 Auditory Competence in Early Life. University Park Press, Baltimore

28. Schneider BA, Trehub SE, Bull D 1979 The development of basic auditory processes in infants. Can J Psychol 33:306-319

29. Engel R, Young NB 1969 Calibrated pure tone audiograms in normal neonates based on evoked electroencephalographic responses. Neuropaediatrics $1: 149-160$

30. Parisi T, Ison JR 1979 Development of the acoustic startle response in the rat ontogenetic changes in the magnitude of inhibition by prepulse stimulation. Dev Psychobiol 12:219-230

31. Davis M, Gendelman DS, Tischler MD, Gendelman PM 1982 A primary acoustic startle circuit: lesion and stimulation studies. J Neurosci 2:791-805

32. Leitner DS, Powers AS, Stitt CL, Hoffman HS 1980 The neural substrate of the startle response. Physiol Behav 25:291-297

33. Strominger NC 1973 The origins, course and distribution of the dorsal and intermediate acoustic striate in the rhesus monkey. J Comp Neurol 147:209234

34. Leitner DS, Powers AS, Stitt CL, Hoffman HS 1981 Midbrain reticular formation involvement in the inhibition of acoustic startle. Physiol Behav 26:259-268 